\title{
Fallbearbeitung nach SGB II - Beobachtungen aus dem Inneren der "black box"
}

\author{
Volker Baethge-Kinsky \\ Peter Bartelheimer \\ Jutta Henke
}

\begin{abstract}
„Moderne Dienstleistungen am Arbeitsmarkt“ - diesen Anspruch der neuen Arbeitsmarktgesetze soll die Grundsicherung für Arbeitsuchende durch integrierte Beratung, Betreuung und materielle Absicherung erfüllen. Die empirische Arbeitsmarktforschung hat bei der Evaluation der Arbeitsmarktpolitik in den letzten Jahren enorme Fortschritte gemacht. Was aber das besondere Profil der Dienstleistung ausmacht, die im Rahmen von „Hartz IV“ Gestalt annimmt, blieb unklar - denn nach wie vor bilden die Leistungsprozesse für die Forschung eine „black box“. Dieser Beitrag riskiert einen Blick auf konkrete Interaktionen zwischen Fachkräften und Arbeitsuchenden: In einer Pilotstudie wurde die Bearbeitung von 20 Fällen bei drei Grundsicherungsträgern über sechs Monate hinweg direkt und umfassend beobachtet.
\end{abstract}

\section{Dienstleistungen am Arbeitsmarkt sind untererforscht}

Dass Dienstleistungen am Arbeitsmarkt untererforscht sind, konnte vermutlich gerade deshalb lange übersehen werden, weil die Forschungslücke so groß ist. Moderne Dienstleistungen am Arbeitsmarkt versprach die „Hartz-Kommission“. Die „Hartz-Gesetze“ führten das Dienstleistungsversprechen im Namen. Und an der reorganisierten Hochschule der Bundesagentur für Arbeit soll man künftig einen Bachelor in "Arbeitsmarktmanagement" und in „Beschäftigungsorientierter Beratung und Fallmanagement" erwerben können - es wird also durchaus erkannt, dass sich in den Begegnungen zwischen Fachkräften und Adressaten ,entscheidet (...), was an Sozialstaat überlebt und wie gesellschaftsverträglich der Modernisierungsprozess gelingt" (Göckler 2006, S. 41). Doch für die empirische Arbeitsmarktforschung blieb das Kerngeschäft der Arbeitsvermittlung bislang eine „black box“, über die vor allem Mutmaßungen und Idealvorstellungen im Umlauf sind: „Was ist ein guter Vermittlungsprozess? Welche Dienstleistung brauchen die Kunden wirklich?" (Hugh Mosley, nach Zeeb 2006, S. 120)

Um diesen Fragen nachzugehen, muss die arbeitsmarktpolitische Wirkungsforschung die Perspektive erweitern. Bislang konzentriert sie sich entweder auf das Geschehen vor der eigentlichen Dienstleistungserbringung oder auf statistisch erfass- te Wirkungen, die nach der Leistungserbringung einzelnen Instrumenten oder Unterschieden zwischen Trägern zugerechnet werden sollen. Organisationsstudien und Implementationsanalysen fragen nach der Umsetzung des politischen Auftrags in Geschäftsmodelle und Organisationsabläufe und messen „Inputs“ (z. B. Aktivitäten der Fachkräfte oder eingesetzte Eingliederungsmittel). Wirkungsanalysen dagegen messen „Outcomes“, wie etwa Übergänge in Beschäftigung, und versuchen, Effekte veränderter Organisationsabläufe auf die beobachtete Dauer der Arbeitslosigkeit oder auf Übergangschancen statistisch zu isolieren.

Was aber geschieht dazwischen - in den Interaktionen zwischen den Adressatinnen oder Adressaten und den Fachkräften der Arbeitsmarktpolitik? Was macht die Qualität einer arbeitsmarktnahen sozialen Dienstleistung nach dem Sozialgesetzbuch II $^{1}$ aus? Wie gelingt es, Beratung, Vermittlung und materielle Sicherung in einer Leistung zu integrieren? Was geschieht, wenn Leistungsberechtigte nach Antragstellung ihre Vermittlerin oder ihren Fallmanager treffen? Wie wirken die Verfahrensvorgaben der Grundsicherungsträger? Was trägt die „Aktivierung“ dazu bei, Ziele am Arbeitsmarkt und in der Lebensführung zu erreichen?

In dieser Perspektive bilden die Organisationsbedingungen der Arbeitsagenturen oder der Grundsicherungsträger nur eine von mehreren institutionell vorgegebenen „Setting“-Bedingungen. Weder durch die "Geschäftsmodelle" der Träger noch durch gesetzliche und politische Vorgaben oder gesellschaftliche Diskurse wird die tatsächliche Dienstleistung vollständig bestimmt. Sie hängt auch von den Einstellungen und Strategien der Menschen ab, die in diesem Rahmen zusammenarbeiten. Erst die Beobachtung des eigentlichen Leistungsprozesses kann daher die „Dienstleistungslücke“ in der Forschung schließen und klären, ob und wie stark gesetzliche Vorgaben und organisatorische Bedingungen die Dienstleistungsarbeit prägen, das Zustandekommen einer Dienstleistungs-

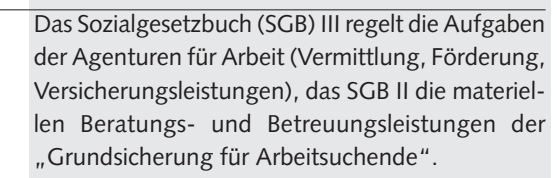
der Agenturen für Arbeit (Vermittlung, Förderung, Versicherungsleistungen), das SGB II die materiellen Beratungs- und Betreuungsleistungen der "Grundsicherung für Arbeitsuchende".

Volker Baethge-Kinsky, Dr., wissenschaftlicher Mitarbeiter des Soziologischen Forschungsinstituts (SOFI), Göttingen. Arbeitsschwerpunkte: Bildungsforschung, Arbeitsforschung, Arbeitsmarktforschung. e-mail: volker.baethge-kinsky@sofi.unigoettingen.de

Peter Bartelheimer, Dr., wissenschaftlicher Mitarbeiter des Soziologischen Forschungsinstituts (SOFI), Göttingen.

Arbeitsschwerpunkte: Sozioökonomische Berichterstattung, Arbeitsmarktforschung. e-mail: peter.bartelheimer@sofi.unigoettingen.de

Jutta Henke, Dipl.-Sozialpädagogin, Projektmitarbeiterin des Soziologischen Forschungsinstituts (SOFI), Göttingen. Leiterin einer Einrichtung der Wohnungslosenhilfe.

e-mail: HenkeBergmans@aol.com 


\begin{tabular}{|c|c|c|}
\hline \multicolumn{3}{|c|}{ Übersicht 1: Einbezogene Standorte } \\
\hline Standort X & Standort Y & Standort Z \\
\hline & Status der Kommune & \\
\hline Kreisfreies Oberzentrum & Kreisangehörige Stadt & $\begin{array}{l}\text { Kreisangehörige } \\
\text { Sonderstatusstadt }\end{array}$ \\
\hline \multicolumn{3}{|c|}{ Agenturbezirk (Vergleichstypen 2005) } \\
\hline $\begin{array}{l}\text { Regionaltyp IIlb (Bezirke mit } \\
\text { durchschnittlicher Arbeits- } \\
\text { losigkeit) }\end{array}$ & $\begin{array}{l}\text { Regionaltyp la (Bezirke in Ost- } \\
\text { deutschland mit schlechtesten } \\
\text { Arbeitsmarktbedingungen) }\end{array}$ & $\begin{array}{l}\text { Regionaltyp Illa (Bezirke mit } \\
\text { überdurchschnittlicher } \\
\text { Arbeitslosigkeit, aber mäßiger } \\
\text { Dynamik) }\end{array}$ \\
\hline \multicolumn{3}{|c|}{ Trägerschaftsmodell } \\
\hline $\begin{array}{l}\text { Arbeitsgemeinschaft nach } \\
\text { \& 44b SGB II }\end{array}$ & $\begin{array}{c}\text { Arbeitsgemeinschaft nach } \\
\text { \& 44b SGB II }\end{array}$ & $\begin{array}{l}\text { zugelassener kommunaler } \\
\text { Träger nach § 6a SGB II }\end{array}$ \\
\hline \multicolumn{3}{|c|}{ Arbeitslosenquote1) Jahresdurchschnitt 2005} \\
\hline $15,3 \%$ & $20,4 \%$ (Landkreis) & 13,0\% (Landkreis) \\
\hline \multicolumn{3}{|c|}{$\begin{array}{l}\text { svp }=\text { Sozialversicherungspflichtige Beschäftigung. } \\
\text { 1) Bezogen auf alle Erwerbspersonen (Berechnung auf Kreisebene). } \\
\text { Quelle: Blien/Hirschenauer 2005; Wolf 2006; Bundesagentur für Arbeit, Statistik. }\end{array}$} \\
\hline
\end{tabular}

beziehung begünstigen, oder wo diese möglicherweise nur im Konflikt mit dem Setting verwirklicht werden kann. Wer wissen will, was in den Leistungsprozessen nach dem SGB II geschieht, kommt nicht umhin, diese auch mit qualitativen Verfahren zu beobachten.

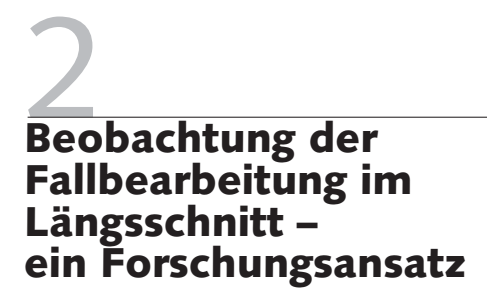

Von Januar bis Juni 2006 führte das Soziologische Forschungsinstitut (SOFI) an der Georg-August-Universität in Göttingen im Auftrag des Instituts für Arbeitsmarkt- und Berufsforschung (IAB) der Bundesagentur für Arbeit eine Konzeptstudie durch, in der bei drei Trägern der Grundsicherung für Arbeitsuchende die Fallbearbeitung in einer begrenzten Zahl von Fällen über einen Zeitraum von sechs Monaten möglichst vollständig $\mathrm{zu}$ beobachten war. $^{2}$ Auch wenn für die Auswahl der drei Standorte forschungspraktische Überlegungen, wie die räumliche Nähe zu den beteiligten Beobachtungsteams, wichtig waren, gelang es mit den drei beobachteten Organisationseinheiten (im Folgenden X, Y, Z genannt), sowohl die beiden wichtigsten Formen der Aufgabenwahrnehmung nach SGB II - Arbeitsgemeinschaft (ARGE) und kommunale Trägerschaft ${ }^{3}$ - wie auch Unterschiede in den regionalen Arbeitsmarktbedingungen (etwa zwischen West- und Ostdeutschland) abzubilden (Übersicht 1).

Bewusst wurde der Beobachtungsgegenstand „Fallbearbeitung " weit, empirisch offen und ohne Bezug auf mehr oder weni-
Bedarfsgemeinschaft einbezogen wurde. Hospitationen in den Organisationseinheiten und Interviews mit Leitungskräften und Experten ergänzten die fallbezogenen Informationszugänge.

Mit den 21 Fachkräften, die in die Beobachtung einbezogen waren, nahmen alle bei SGB-II-Trägern üblichen Geschäftsbereiche der "Aktivierung“ an der Untersuchung teil, das heißt: Vermittlung oder Fallmanagement für junge Erwachsene unter 25 Jahren wie auch für Erwachsene bzw. für Ältere („,50 plus“). ${ }^{5}$ In X gelang es zusätzlich, drei Fachkräfte der Leistungssachbearbeitung einzubeziehen. Einzelne Vorsprachen nicht gerechnet, wurden in X, Y und Z 20 Fälle mit unterschiedlichen Haushaltskonstellationen beobachtet (Übersicht 1). Weil die Träger X und Z immer noch damit beschäftigt waren, den Anfang 2005 beim Übergang zum SGB II übernommenen Fallbestand ,abzuarbeiten", handelte es sich nur bei vier Adressaten um echte „Neufälle“. In elf anderen Fällen bezogen die Adressaten bereits seit 2005 Leistungen, erhielten aber erst Anfang 2006 einen ersten Beratungstermin in der „Aktivierung“. In der ostdeutschen ARGE Y kam hinzu, dass sich Neuzugänge junger gen (Dunkel et al. 2004).

Ohne die freiwillige Bereitschaft der drei einbezogenen SGB-II-Träger, aber vor allem vieler Fachkräfte und Adressaten, sich ,in die Karten“ und über die Schulter schauen zu lassen, und ohne ihre nachsichtige Duldung der Tatsache, durch eines von drei örtlichen Forscherteams ,auf Schritt und Tritt" begleitet zu werden, wäre eine Fallbeobachtung in der gewünschten Intensität und Reichweite nicht möglich gewesen. Denn das Beobachtungsprogramm sah den Versuch vor, den ganzen Leistungsprozess in den Blick zu bekommen. Dies bedeutete vor allem, möglichst alle Gesprächstermine („Vorsprachen“) der Adressaten bei ihrem „persönlichen Ansprechpartner“ (pAp) zu beobachten und aufzuzeichnen sowie nach jeder Vorsprache mit beiden Beteiligten Nachgespräche zu führen. Ergaben sich Verabredungen, wie eine Vermittlung in die Schuldnerberatung oder eine Maßnahmezuweisung, wurden die Gesprächstermine dort - sofern die Beteiligten zustimmten ebenfalls begleitet. Einzelfallbezogen steuerten weitere Fallbeteiligte ihre jeweilige Sicht bei. Mit Fachkräften und Adressaten wurden teilstandardisierte Interviews geführt, wobei nach Möglichkeit die ganze
2 Auch wenn es im Folgenden nur um Leistungen der Grundsicherung geht, lässt sich der Forschungsansatz auf Dienstleistungen der Arbeitsagenturen im Regelkreis des SGB III übertragen. Für die ausführliche Dokumentation sei auf den umfangreichen Projektbericht verwiesen (BaethgeKinsky et al. 2006).

3 Nach dem SGB II sind drei Trägerkonstruktionen möglich: Der "Normalfall" ist die Arbeitsgemeinschaft nach § 44b SGB II (ARGE), in der Kommune und örtlich zuständige Agentur für Arbeit unter einem einheitlichen organisatorischen Dach die Aufgaben im Rahmen der Grundsicherung übernehmen. Bei den nach § 6a SGB II zugelassenen kommunalen Trägern erfüllt die Kommune - bislang befristet - alle Aufgaben der Grundsicherung und Vermittlung. In den wenigen Fällen "getrennter Aufgabenwahrnehmung " kam es nicht zur Bildung einer ARGE bzw. wurde diese wieder aufgelöst.

4 Zu Standards von Fallmanagement vgl. z. B. Autorenteam 2004; Kolbe/Reis 2005. Auch aus Sicht des zuständigen Ministeriums bilden alle „Elemente des SGB-II-Leistungsrechts" zusammen den "Aktivierungsprozess": "arbeitsmarktpolitische Instrumente, flankierende Maßnahmen nach $\S 16$ Abs. 2 Satz 2 Nr. 1 bis 4, Anreize und Sanktionen, aber durchaus auch die sogenannten, passiven Leistungen'“ (BMWA 2005, S. 2).

5 Wegen des beschränkten Umfangs der Untersuchung konnten jedoch noch nicht alle organisatorisch unterschiedlichen Pfade der Fallbearbeitung an jedem Standort systematisch untereinander verglichen werden. 
Erwachsener auf das Ende eines Schuljahrs konzentrieren und die Fluktuation mangels Vermittlungschancen gering war.

Insgesamt wurden 53 Vorsprachen beobachtet und transkribiert. Zum Gesprächsmaterial zählen außerdem 73 Nachbefragungen, sieben Gespräche mit Fallbeteiligten und 52 leitfadengestützte Interviews. Die Analyse der beobachteten Gespräche ergab einen fortlaufend ergänzten Katalog charakteristischer Gesprächsereignisse („Sequenzen“). Auf dieser Grundlage wurde für jeden Fall ein Beobachtungsprotokoll erstellt, das den Verlauf der Fallbearbeitung nachzeichnet. Gleichzeitig wurden im Gesprächsmaterial Beispiele und Belege für grundlegende Probleme der Fallbearbeitung identifiziert. Ergänzende Organisationsbeschreibungen der drei Grundsicherungsträger stützen sich sowohl auf eigene Beobachtung wie auf Interviews mit Fachkräften.

Im beschränkten Rahmen einer Konzeptstudie mit kurzer Laufzeit konnten weder regionale und organisatorische Varianten der Fallbearbeitung noch die Vielfalt sozioökonomischer Fallkonstellationen ausreichend erfasst werden. Doch lassen sich bereits zentrale Setting-Merkmale und Problemzonen der Fallbearbeitung erkennen, von denen das besondere Profil der Dienstleistungsarbeit bei den SGB-II-Trägern abhängt. Zum ersten Mal entstand ein dichtes Bild des sozialen Handelns von Fachkräften und Adressaten im gesetzlichen Rahmen der Grundsicherung für Arbeitsuchende.

\section{Probleme der Fallbearbeitung}

\subsection{PERSÖNLICHER AUFTRAG UNTER BEDINGUNGEN VON ASYMMETRIE}

Bei jeder personenbezogenen Dienstleistung müssen Fachkräfte und Adressaten zusammenwirken: ${ }^{6}$ Sie müssen Gegenstand und Ziel der Leistungen aushandeln und ihre Handlungen abstimmen. $\mathrm{Ob}$ im Rahmen der Fallbearbeitung überhaupt Beratung, Betreuung oder Vermittlung zustande kommt oder ob es beim einseitigen Verwaltungshandeln bleibt, hängt von einem personalisierten Auftrag ab, den nur die Adressaten erteilen können. In der Grundsicherung muss dieser persönliche Auftrag - anders als bei freiwilligen Dienstleistungsangeboten oder bei Adressaten

\begin{tabular}{|c|c|c|c|c|}
\hline & $x$ & $\mathbf{Y}$ & $\mathbf{Z}$ & Gesamt \\
\hline \multicolumn{5}{|l|}{ Einbezogene Fachkräfte } \\
\hline Für unter 25-Jährige & 2 & 3 & 1 & 6 \\
\hline $\begin{array}{l}\text { davon Vermittlung } \\
\text { davon Fallmanagement }\end{array}$ & $\begin{array}{l}1 \\
1\end{array}$ & 3 & 1 & \\
\hline Für über 25-Jährige & 1 & 1 & 5 & 7 \\
\hline $\begin{array}{l}\text { davon Vermittlung } \\
\text { davon Fallmanagement }\end{array}$ & 1 & 1 & 5 & \\
\hline Für über 50-Jährige & & & 2 & 2 \\
\hline Arbeitgeberservice & 1 & & 1 & 2 \\
\hline Passive Leistungen & 3 & 1 & & 4 \\
\hline Insgesamt & 7 & 5 & 9 & 21 \\
\hline \multicolumn{5}{|l|}{ Einbezogene AdressatInnen } \\
\hline $\begin{array}{l}\text { Weiblich } \\
\text { Männlich }\end{array}$ & $\begin{array}{l}4 \\
4\end{array}$ & $\begin{array}{l}1 \\
4\end{array}$ & $\begin{array}{l}4 \\
3\end{array}$ & $\begin{array}{r}9 \\
11\end{array}$ \\
\hline Insgesamt & 8 & 5 & 7 & 20 \\
\hline $\begin{array}{l}\text { davon Alleinlebend } \\
\text { davon Allein erziehend }\end{array}$ & $\begin{array}{l}3 \\
1\end{array}$ & & $\begin{array}{l}3 \\
2\end{array}$ & $\begin{array}{l}6 \\
4\end{array}$ \\
\hline Migrationshintergrund & 3 & & 1 & 4 \\
\hline Unter 25 Jahre & 4 & 4 & 1 & 9 \\
\hline 25-49 Jahre & 3 & 1 & 4 & 8 \\
\hline Älter als 50 Jahre & 1 & & 2 & 3 \\
\hline
\end{tabular}

mit Wunsch- und Wahlrechten - in einem vom Gesetzgeber vorgegebenen asymmetrischen Setting ausgehandelt werden. Die soziale Dienstleistung der „Information, Beratung und umfassende(n) Unterstützung durch einen persönlichen Ansprechpartner" ( $\$ 4$ Abs. 1 Nr. 1 SGB II) steht in einem unlösbaren Verfahrenszusammenhang mit den Geldleistungen zum Lebensunterhalt. Einladungen zum Gespräch, der Abschluss einer Eingliederungsvereinbarung (nach $\S 15$ SGB II), etwaige Auflagen oder Vermittlungsentscheidungen sind für die Adressaten verpflichtend. Versäumnisse oder Weigerung führen zur Absenkung oder zum Wegfall der existenzsichernden Leistungen. Dass der Gesetzgeber den Fachkräften mit dem Grundsatz des „Forderns und Förderns" in Aussicht stellt, Mitwirkung erzwingen zu können (Reis 2006, S. 196), ändert nichts daran, dass sie zur Erfüllung ihrer Aufgaben den persönlichen Beratungs-, Vermittlungs- oder Betreuungsauftrag der Adressatinnen und Adressaten benötigen. Die Folge ist, dass beide Seiten informelle Spielräume für die notwendigen Aushandlungen nutzen müssen und dass die Adressaten dies im „Wissen um die Sanktionsgewalt (ihres) Gegenübers" tun (Autorenteam 2004, S. 15).

\subsection{GESPRÄCHSTERMINE: ZEIT FÜR DIENSTLEISTUNG}

Auch wenn ein Teil der Dienstleistung „Fallbearbeitung“ - wie die Zusendung von Stellenangeboten, die Dokumentation oder Antragsbearbeitung - ohne persönlichen Kontakt auskommt, entscheidet sich die Qualität der Dienstleistung in den persönlichen Gesprächen zwischen Adressaten und Fachkraft. Von der Zeit, die hierfür zur Verfügung steht, hängt ab, ob überhaupt eine komplexere („verstehende“ oder „diffusere“, vgl. Harrach et al. 2000, S. 88ff.) Dienstleistungsbeziehung entstehen kann. Die Kontaktdichte, das heißt die Dauer der Vorsprachen und die Abstände zwischen ihnen, bildet daher eine wichtige organisatorische Setting-Bedingung. Sie konnte in der Studie nur für die Fachkräfte in der „Aktivierung“ erfasst werden.

In zwei Dritteln der Fälle fanden im Beobachtungszeitraum (9. Januar bis 19. Juni 2006) durchschnittlich drei bis vier Vorsprachen statt. Das Spektrum reichte vom Fall eines jungen Mannes, bei dem es aus Krankheitsgründen nicht zum Gesprächstermin kam, bis zum Fall eines Migranten mit sechs Vorsprachen in drei Monaten. Einmal lag zwischen dem Erst- und dem Folgegespräch nur ein Tag, in einem anderen Fall waren es 71 Tage. Das kürzeste beobachtete Gespräch dauerte sechs, das längste 74 Minuten. 
Die Unterschiede in den beobachteten Zeitmustern erklären sich weniger aus den Betreuungsrelationen (Fallzahlen je Fachkraft), als vielmehr aus anderen organisatorischen Gegebenheiten. So kam es in X und $\mathrm{Z}$ im Durchschnitt pro Fall etwa alle sechs Wochen zu einer Vorsprache, die einzelnen Gespräche dauerten durchschnittlich 42 bzw. 43 Minuten. Der Träger X war bemüht, Gespräche in regelmäßigen Abständen (durchschnittlich alle sechs Wochen) sicherzustellen, wobei Erstgespräche mit Eingliederungsvereinbarung etwa eine Stunde in Anspruch nahmen, Folgegespräche im Durchschnitt nur 36 Minuten. In $\mathrm{Z}$ dagegen waren die Folgegespräche eher zeitaufwändiger als die Erstgespräche, da eine Eingliederungsvereinbarung hier frühestens im Zweitgespräch abgeschlossen wurde. In Y dagegen kam es pro Fall nur etwa alle zehn Wochen zu einem persönlichen Gespräch, das mit durchschnittlich 17 Minuten nur knapp halb so lang dauerte wie an den anderen Standorten. Hier waren in der ARGE vor allem Bewerbungen nachzuweisen, während ein größerer Teil der individualisierten Fallarbeit in Maßnahmen stattfand.

In immerhin sechs von 20 Fällen kam wenigstens ein Termin auf Initiative der Adressaten zustande. Viele Fachkräfte legen Wert darauf, solche spontanen Vorsprachen zu ermöglichen - im Gegensatz zu den Leistungssachbearbeitern, die ,ausschließlich auf Termin " arbeiten und daher für die kurzfristige Klärung von Leistungsfragen kaum erreichbar sind.

Die beobachtete Kontaktdichte, die wenigstens für die Fachkräfte in $\mathrm{X}$ und $\mathrm{Z}$ die Grenze des Realisierbaren markieren dürfte, ist ein Argument dafür, die Ziele und Erwartungen an Vermittlung oder Fallmanagement realistisch zu beschränken. Was sich im Leben der Adressaten an Unvorhergesehenem und Ungeplantem ereignet, muss in einen begrenzt flexiblen Zeitrahmen eingepasst und im Durchschnitt in einer Vorsprache alle sechs bis zehn Wochen besprochen und bearbeitet werden. Zweifellos steht den „persönlichen Ansprechpartnern" damit mehr Zeit zur Verfügung als den Fachkräften in den Arbeitsagenturen, aber für eine umfassende Betreuung in prekären Lebenslagen reicht dies nicht.

\subsection{INDIVIDUALISIERTE DIENSTLEISTUNG BEI HOHEN FALLZAHLEN?}

Bei einer Leistung, die für mehr als 5 Mio. erwerbsfähige ALG-II-Berechtigte in 3,6 Mio. Bedarfsgemeinschaften erbracht wird, sind Standardisierungen unvermeidlich. Probleme können sich jedoch aus dem gleichzeitigen Ziel des SGB II ergeben, die Erwerbsintegration der „Kundinnen“ und „Kunden" durch individualisierte und passgenaue Dienstleistungen zu optimieren.

Eine Möglichkeit, mit dem Widerspruch zwischen hohen Fallzahlen und individuellem Bearbeitungsauftrag umzugehen, besteht für die Fachkräfte darin, sich auf einen Teil des Fallbestands zu konzentrieren. Weil dies unabhängig von den formalisierten Verfahren der „Kundensegmentierung" in den Organisationseinheiten geschieht, wurden solche Routinen in der Beobachtung als „Nachsegmentieren“ bezeichnet. Waren die Fachkräfte in $\mathrm{X}$ bemüht, dies zu vermeiden und mit allen Adressaten gleich intensiv zu arbeiten, galt es in $\mathrm{Z}$ als akzeptabel und unvermeidlich, Fälle ,auch mal liegen zu lassen“. Der Anteil solcher „Drei-Monats-Kunden“ wurde auf etwa ein Drittel geschätzt.

Bei der ARGE Y wurde die gesamte Fallbearbeitung als eine Verkettung von Routinen interpretiert, was eine Reaktion auf den verschlossenen ostdeutschen Arbeitsmarkt sein könnte. Die beobachteten jungen Erwachsenen mussten monatlich vorsprechen, um Bewerbungen nachzuweisen, und wurden alle drei Monate über Gruppenveranstaltungen einer Maßnahme zugewiesen.

In neun der 20 Fälle wurde der Abschluss wenigstens einer neuen Eingliederungsvereinbarung (EinV) beobachtet. Dieses Verfahrenselement wurde in die Arbeitsmarktpolitik eingeführt, um den Leistungsprozess am Einzelfall auszurichten, und eine Arbeitshilfe (Bundesagentur für Arbeit 2006) betont, dass die EinV ,individuell auf die Person und die vorliegenden Umstände abzustimmen “ ist, um Rechtsgültigkeit zu erlangen. Andererseits soll mit allen Adressatinnen und Adressaten eine Vereinbarung geschlossen werden, und zwar möglichst schon im Erstgespräch. Dass diese Anforderung eher standardisierend als individualisierend wirkt, wurde besonders in $\mathrm{Y}$ beobachtet, wo die Eingliederungsvereinbarung als Verwaltungsroutine bereits vor dem Erstgespräch vorberei- tet und binnen weniger Minuten unterschrieben wurde. In $\mathrm{X}$ und $\mathrm{Z}$ dagegen wurden die Vereinbarungen in den persönlichen Gesprächen gründlich erarbeitet und spiegeln den Gesprächs- bzw. Fallverlauf, wenn auch mit Übersetzungsproblemen.

Die Erfassung der Daten, die sie z. B. für den Abschluss einer Eingliederungsvereinbarung benötigen, stellt die Fachkräfte vor weitere "Standardisierungsprobleme“. In den ARGEn X und Y wurde etwa das Erstellen von Bewerberprofilen im neuen ITVerfahren VerBIS beobachtet. Dies geschah in Y eher formalisiert und verwaltungsmäßig, in X in gründlicher Erörterung mit dem Adressaten. Bei beiden Verfahrensweisen blieben Zweifel, ob diese Profile ein „passgenaues“ Matching ermöglichen; viele Fachkräfte hielten diese Aufgabe nicht für automatisierbar und nutzten daher unabhängig von den IT-Verfahren eigene Abfrageroutinen. Dennoch waren große Zeitspannen innerhalb der Gespräche durch Eingaben in die IT-Verfahren der Träger geprägt; in X konnten stumme „EDV-Pausen“ für Eingaben in den PC (einschließlich der Vorbereitung der Eingliederungsvereinbarung) bis zu einem Drittel der Gesprächszeit ausmachen.

\subsection{AKTIVIERUNG - EINE WACKLIGE HIERARCHIE}

Die Analyse des Gesprächsmaterials liefert Hinweise, dass Gegenstand und Ziel der neuen Dienstleistung vor allem durch eine spezifische Rollenverteilung festgelegt werden. Im Gespräch sitzen sich „Aktivierer“ und „Aktivierte“ gegenüber. Ein Grundproblem der beobachteten Leistungsprozesse im Kontext des SGB II wird daran erkennbar: Die weitreichenden Kompetenzen und Ermessensspielräume, die das Gesetz den Fachkräften zuweist, begründen eine hierarchische Beziehung, die dem Alltagsverständnis von den förderlichen Bedingungen für eine Beratung zuwiderläuft ${ }^{7}$ und die im Gespräch immer wieder herge-

\footnotetext{
Beratungsbeziehungen sind zwar hierarchisch aber die Rollen sind wechselnd verteilt: Die Ratsuchenden erteilen einen Beratungsauftrag und entscheiden später darüber, ob der Rat ihrer Lebenssituation angemessen ist und wie weit sie inn befolgen. In den Phasen dazwischen unterstellen dagegen beide Seiten einen Kompetenzvorsprung des Beraters, der Vorrechte bei der Gesprächsorganisation, wie Fragerechte und Antwortpflichten, begründet.
} 
stellt, befestigt und als Konfliktpotenzial abgemildert werden muss.

Besonders deutlich zeigt sich dieses Problem beim Abschluss der Eingliederungsvereinbarung. Bei dieser Rechtskonstruktion des SGB II handelt es sich um einen asymmetrischen, „hinkenden“ Vertrag (Berlit 2006): Die Adressaten müssen abschließen, und nur sie tragen bei Nichterfüllung ein Sanktionsrisiko. Mit seiner Funktion, als Grundlage für spätere Sanktionen zu dienen, macht dieses Verfahrenselement die Gesprächshierarchie unumkehrbar. Die Fachkräfte entschärfen die Asymmetrie zwar gesprächsweise: Einerseits sprechen sie von einem „Vertrag, den wir beide miteinander abschließen" (eine Fallmanagerin in X). Andererseits zeigen Rechtsbelehrung und Vertragsinhalte, dass diese Gegenseitigkeit eine Fiktion ist. So werden die Verpflichtungen der Fachkräfte eher abstrakt formuliert („Unterstützung bei der Arbeitssuche“), oder es werden die bereits im laufenden Gespräch erbrachten Leistungen angeführt. Die Verpflichtungen der Adressaten werden dagegen detailliert benannt und liegen sämtlich in der $\mathrm{Zu}$ kunft.

Adressaten scheinen sowohl die Rollenzuweisung als auch die Asymmetrie der Beziehung immer dann zu akzeptieren, wenn sie von der Arbeitsmarktkompetenz der Fachkraft überzeugt sind: „Meine Sachbearbeiterin, Frau [Name], die klemmt sich da wirklich dahinter (...), wobei natürlich nicht die ganze Arbeit auf ihren Schultern lasten soll, sondern ich muss mich da auch mitdrehen.“ Als „unschädlich“ erwies sich die vorgegebene Rollenverteilung auch, wenn die Fachkräfte in der Fallbearbeitung Wert darauf legten, die Adressaten bei der Erreichung ihrer selbst gesetzten Ziele reflektierend zu unterstützen.

Überwiegend vertraten die Fachkräfte die Auffassung, dass Mitspracherechte der Adressaten und die kooperative Vereinbarung von Zielen deren Umsetzung begünstigen. Aus dieser Haltung ergab sich in den beobachteten Fällen eine Arbeitsweise, die möglichst lange auf den Einsatz der verfügbaren Druckmittel verzichtete. Sanktionsinstrumente wurden eher hergezeigt als angewandt. Nur in zwei Fällen wurden Sanktionen beobachtet, diese allerdings bedeuteten für die beiden jungen Erwachsenen gleich den Wegfall der Leistung für drei Monate.

Die Dienstleistungsbeziehung geriet dagegen erkennbar unter Druck, wenn
Adressaten die Arbeitsmarktkompetenz der Fachkräfte bezweifelten oder wenn der Weg zur Arbeitsmarktintegration kontrovers blieb. So verlangten die Fachkräfte in 13 der 20 Fälle die Erstellung oder Überarbeitung von Bewerbungsunterlagen und wiesen in neun Fällen Maßnahmen mit Bewerbungstraining zu. Meist aber waren die Adressaten vom Nutzen nicht überzeugt oder bezweifelten, dass sich ihre Arbeitsmarktchancen dadurch erhöhen würden. In fünf Fällen wurden solche Maßnahmen dann auch nicht angetreten, abgebrochen oder beendet, ohne dass Bewerbungsunterlagen erstellt waren.

In wenigstens zehn der beobachteten Fälle widersprach die Rollenverteilung "Aktivierer" und „Aktivierter" - der tatsächlichen Fallkonstellation. In allen vier Fällen, in denen eine erfolgreiche Bewerbung beobachtet werden konnte, hatten die Adressaten die Stelle selbst gefunden. Sogar in einem der beiden Sanktionsfälle würdigt die Vermittlerin ausdrücklich das Engagement der jungen Frau bei der Suche nach einem Ausbildungsplatz - die Sanktion ist hier Folge einer Meinungsverschiedenheit um den Nutzen einer Trainingsmaßnahme. Nicht ein vom gesetzlichen Setting unterstelltes Aktivierungsdefizit führt in den Konflikt, vielmehr misslingt die Aushandlung eines von beiden Seiten getragenen Integrationswegs.

In den Fällen, in denen prekäre persönliche Lebensumstände der Adressaten einer Orientierung auf den ersten Arbeitsmarkt entgegenstanden oder diese nach langer Arbeitslosigkeit resigniert hatten, zeichnete sich eine andere Konfliktkonstellation ab: Das Arbeitsbündnis geriet in Gefahr, weil die „aktivierenden“ Fachkräfte an die Grenzen des Maßnahmeangebots oder des örtlichen Arbeitsmarkts stießen.

\subsection{AKTIVIERUNGSTHEMEN - UND ANDERE}

Das gesetzliche Setting der Grundsicherung lässt eine breitere Themenstruktur zu als der Regelkreis des SGB III in der Arbeitsvermittlung. Nach \$16 SGB II können auch zu arbeitsmarktferneren Problemen Leistungen erbracht werden, sofern diese im Einzelfall für die Arbeitsmarktintegration erforderlich sind. ${ }^{8}$ Welche Themen in der Fallbearbeitung „auf den Tisch“ kommen, hängt sowohl von den Adressaten als auch von den Fachkräften ab. Allerdings entscheiden Letztere darüber, was davon als
Gegenstand der „Aktivierung“ identifiziert wird. Beide Seiten haben Gründe, bestimmte Themen zu vermeiden.

In 13 Fällen wurde die prekäre materielle Situation der Adressatinnen und Adressaten zum Gesprächsgegenstand in der Aktivierung. Fast immer waren es die Ratsuchenden, die ihre Geld- oder Wohnungsprobleme mitbrachten. Diese Problemanmeldungen wurden von Vermittlern und Fallmanagerinnen stets in die Zuständigkeit der Leistungssachbearbeitung zurückverwiesen; nur ausnahmsweise schalteten sie sich selbst ein. Eine Vermittlerin spricht von „Vermeidung“: „Das ist eine Komponente, wo ich merke, dass sie mich frustriert. Also, wenn ich das so nah an mich rankommen lasse." Eine andere würde am liebsten ihr „Portemonnaie herausholen“.

Die Adressaten verteidigen verbleibende Entscheidungsspielräume darüber, welche Themen in die Fallbearbeitung gehören und welche nicht. Im Fall einer nichtehelichen Lebensgemeinschaft erschien es der Adressatin als übergriffig, dass die Vermittlerin die Verteilung der Verdienerrolle und der Kinderbetreuung in deren Beziehung ansprach. Der ostdeutsche Landmaschinenschlosser will weder Energieschulden noch das Alkoholproblem, das sein Fallmanager bei ihm vermutet, verhandelt wissen. Ihn stört an „Hartz IV“ vor allem das „drüber Reden, Reden, Reden“. Auch der verschuldete Kraftfahrer findet, persönliche Angelegenheiten gingen seine Fallmanagerin, die er als Arbeitsmarktexpertin sieht, nichts an: „Also private Sachen sollte man rauslassen."

Beobachtet wurde aber auch, dass Fachkräfte Probleme im persönlichen Bereich ausdrücklich außerhalb des Aktivierungsprozesses stellen, um sie überhaupt behandeln zu können. Sie wollen ihre Bearbeitung nicht in eine Eingliederungsvereinbarung einschließen, wo sie zugleich verpflichtend und trägerintern zugänglich formuliert würden. Der suchtkranke Programmierer spricht mit seiner Fallmanagerin von Anfang an offen über sein Alkoholproblem, und sie stellt von Anfang an klar, dass ihre Hilfeangebote hierzu freiwillig sind.

8 Das zuständige Bundesministerium sieht "soziale Stabilisierung " neben Integration in ungeförderte Beschäftigung und Erhalt bzw. Verbesserung der Beschäftigungsfähigkeit als eigenständig zu bewertende Ziele des SGB II (BMWA 2005, S. 6f.). 


\subsection{LEISTUNGEN AUS EINER HAND?}

Das SGB II sieht für eine komplexe, arbeitsteilig organisierte Dienstleistung einen persönlichen Ansprechpartner (pAp) vor. Dieser kann Aufgaben delegieren, soll aber das Dienstleistungsversprechen eines ,integrierten System(s) von Beratung, Betreuung und materieller Absicherung" realisieren. Die beobachteten Leistungsprozesse erwiesen sich jedoch als eher fragmentiert und segmentiert; "Aktivierung“ wurde als spezialisierte Leistung in strikter Arbeitsteilung zu Sachbearbeitung, Außendienst und beauftragten Dritten aufgefasst.

Wie die große Mehrzahl der Grundsicherungsträger hatten sich die Standorte $\mathrm{X}$, Y und Z entschieden, die Gewährung ,aktivierender" Eingliederungsleistungen und die „passiver" Geldleistungen organisatorisch getrennten Geschäftsbereichen zu übertragen. In Leistungsfragen verweisen die „aktivierenden“ Fachkräfte nach dieser Logik an die Sachbearbeiter. Doch diese sind nicht nur wegen ihrer Arbeitsbelastung schwer erreichbar für die Adressaten, nach ihrem Selbstverständnis gehört die ganzheitliche „Beratung“ auch zu den Aufgaben der Fachkräfte in Vermittlung und Fallmanagement. Wenn sich jedoch keiner der beiden Bereiche für die Beratung zu Geldleistungen zuständig erklärt, was einige Fallverläufe nahelegen, gefährdet die Arbeitsteilung den Anspruch der Adressaten auf eine Leistungsberatung. Da beide Bereiche zudem auf der Grundlage unterschiedlichen Fallwissens arbeiteten, kam es auch zu anderen Bearbeitungskonflikten: Im Fall eines Asylbewerbers blieb der Leistungsanspruch so lange unklar, dass eine geplante Maßnahme nicht durchgeführt werden konnte. Bei einer ehemals selbstständigen Gastwirtin drängte die Sachbearbeiterin auf Umzug, die Fallmanagerin auf ein Bewerbungstraining - an dem die Adressatin dann nicht teilnahm.

Bei der Beauftragung Dritter (Maßnahmeträger) kam es dann zu Problemen, wenn die Fachkräfte Adressaten auf der Grundlage unvollständiger Informationen in eine Maßnahme zuwiesen. Dies führte in zwei Fällen später dazu, dass die Fallmanager dem Abbruch einer Trainingsmaßnahme zustimmten. Oder sie gingen mangels Rückmeldung fälschlich davon aus, dass die spezialisierten Dritten das Problem bearbeiteten. In einem Fall erfuhr die Fallmanagerin erst als der Familie die Wohnung bereits fristlos gekündigt war, dass der Adressat nicht in der Schuldnerberatung angekommen war, und auch die Verweisung an die Sachbearbeiterin (die den Mietrückstand durch Anrechnung überzahlter Leistungen unwissentlich verschärfte) und an die kommunale Wohnungsfachstelle (die wieder an die Schuldnerberatung verwies) führte nicht zur Problembearbeitung.

Zwischen den Grundsicherungsträgern und der Agentur für Arbeit waren die „Schnittstellenprobleme“ nicht geringer als gegenüber beauftragten Maßnahmeträgern. Vor allem gegenüber jungen Erwachsenen hatten Berufsberatung und „Aktivierung" einen konkurrierenden Auftrag, die Adressaten wurden doppelt betreut. Mehrfach rechnete der SGB-II-Träger aber auch Kindergeldzahlungen an, die die Adressaten nicht erhielten, z. B., weil sie bei der Berufsberatung keinen Termin bekamen. Bei einer älteren Frau in Z fehlte dem Fallmanager die - noch im Arbeitsamt unterschriebene - Erklärung über erleichterten Bezug von Arbeitslosenhilfe ( $\$ 428$ SGB III) und damit die Entscheidungsgrundlage, ob sie überhaupt ein „Aktivierungsfall“ für ihn war.

\subsection{UNSICHERE RECHTSFORMEN}

Im rechtlichen Setting des SGB II besteht für die Fachkräfte bei Beratung, Vermittlung und Fallmanagement ein breiter Ermessensspielraum; die Ergebnisse haben jedoch leistungsrechtliche Konsequenzen und müssen rechtssicher sein. Dies hat widersprüchliche Auswirkungen. Einerseits wird die Fallbearbeitung vor allem durch die Eingliederungsvereinbarung ,verrechtlicht". Andere Beobachtungen deuten dagegen auf einen Verlust an Rechtsförmigkeit hin. So liegt die Gewährung von Eingliederungsleistungen ganz im Ermessen der Fachkräfte und wird zum Gegenstand gesprächsweiser Aushandlung - sie lassen sich weder beantragen, noch können Rechtsmittel gegen eine ablehnende Entscheidung eingelegt werden. Da aber auch in Vermittlung oder Fallmanagement noch Leistungen beantragt werden müssen (etwa die Erstattung von Bewerbungskosten), müssen die Adressaten mit beiden Vorgehensweisen umgehen können. Für sie ist schwer überschaubar, wer entscheidet, wann Entscheidungen fallen und welche rechtlichen Bedingungen und Konsequenzen in der konkreten Interaktion relevant sind. Selbst der Rechtscharakter der Ein- gliederungsvereinbarung wird möglicherweise erst im Konfliktfall deutlich, da diese von den Fachkräften oft konfliktvermeidend - z. B. als „Protokoll“ - ins Gespräch eingeführt wird. In beiden beobachteten Sanktionsgesprächen war der Verfahrensschritt „Anhörung“ nicht als solcher erkennbar oder er folgte auf die Sanktionsentscheidung. Viele Vorsprachen erhalten so einen unklaren rechtlichen „Subtext“.

\subsection{FALL ODER FALLBETEILIGT - DIE BEDARFSGEMEINSCHAFT}

Die Aktivierungsdienstleistung richtet sich an einzelne Erwerbsfähige; ihr materieller Leistungsanspruch und ihre Verfügbarkeit für den Arbeitsmarkt entscheiden sich aber in der Bedarfs- bzw. in der Haushaltsgemeinschaft. Ausdrücklich wird sie im SGB II - anders als dies im früheren BSHG der Fall war - als Adressatin des Aktivierungsprozesses genannt ( $\$ 1$ Abs. 1 Satz 1 und $\$ 14$ Satz 2 SGB II). „Soweit Anhaltspunkte nicht entgegenstehen“, wird vermutet, dass ein Antragsteller die gesamte Bedarfsgemeinschaft vertritt ( $\$ 15$ Abs. 2).

In den beobachteten Organisationseinheiten setzten die Verfahren der „Kundensegmentierung" zunächst immer an Einzelpersonen an, ohne die Bedarfsgemeinschaft zu berücksichtigen. Die erwerbsfähigen Hilfebedürftigen einer Bedarfsgemeinschaft hatten daher unter Umständen unterschiedliche Ansprechpersonen, und es hing von den Fachkräften ab, wer als vorrangig zu ,aktivieren“ galt und ob Angehörige einbezogen wurden. In dieser zentralen Frage schien erhebliche fachliche Unsicherheit zu herrschen. In X wurde fast ausschließlich am Einzelfall gearbeitet, in $\mathrm{Z}$ bemühen sich die Vermittlerinnen eher, Partner und Partnerin einzubeziehen. Wer in Haushalten mit Kindern für deren Betreuung „zuständig“ sein sollte, wurde in einigen Fällen angesprochen, in anderen wurde vorausgesetzt, dass die Partnerin diese Aufgabe übernimmt. Auf den Widerstand der AdressatInnen traf die Thematisierung in einem Fall, in dem die Vermittlerin die Geschlechterarrangements und die Verteilung von Haus- und Erwerbsarbeit infrage stellte. In Z wurde jedoch auch eine Fallkonferenz mit dem Ziel beobachtet, die Fallbearbeitung zusammenzuführen. 


\section{1 \\ Erste Antworten und neue Fragen}

Es war nicht Aufgabe der Konzeptstudie, das beobachtete Fallgeschehen zu bewerten oder Empfehlungen zur Ausgestaltung der Leistungsprozesse zu geben. Aus dem Material ergeben sich jedoch sowohl Schlussfolgerungen zum Charakter der Dienstleistungsarbeit als auch Fragen für weitere Recherchen und Analysen.

\subsection{GESETZLICHE VORGABEN UND ORGANISATORISCHE BEDINGUNGEN}

Bei allen Unterschieden in den Geschäftsmodellen der drei beobachteten Organisationseinheiten bleibt doch der bestimmende Eindruck, dass in dem neuen Leistungssystem eine charakteristische Dienstleistung entsteht, eben „Fallbearbeitung nach SGB II", die sich bei allen organisatorischen und örtlichen Unterschieden von anderen sozialen Dienstleistungen abhebt. Wirkungsforschung muss daher nicht nur die „Implementation“, sondern auch das gesetzliche Setting selbst zum Gegenstand machen: etwa den „Aktivierungsauftrag“ oder die Regelungen zur Eingliederungsvereinbarung. Andererseits müsste die Beobachtung abweichender organisatorischer Lösungen (etwa der integrierten Leistungssachbearbeitung) zeigen, welche der festgestellten Probleme durch eine andere Umsetzungspraxis im gegebenen gesetzlichen Rahmen lösbar sind.

\subsection{AUF STEUERUNGS- UND KONTROLLANSPRÜCHE VERZICHTEN}

Soziale Dienstleistungen brauchen einen personalisierten Auftrag. Diese Bedingung wird um so wichtiger, je komplexer und "diffuser" der organisatorische Auftrag ist. Sie lässt sich missachten, aber nicht aufheben: Wird sie im „Setting“ der Dienstleistung nicht hinreichend berücksichtigt, setzt sie sich hinter dem Rücken der Organisation durch. Für die Aushandlung des Auftrags und der Dienstleistungsbeziehung nutzen oder schaffen die Beteiligten dann informelle Spielräume. Für die weitere Ausgestaltung der Fallbearbeitung nach SGB II könnte dieses Ergebnis die Konsequenz haben, dass die Organisationseinheiten Spielräume akzeptieren und auf
Steuerungs- und Kontrollansprüche verzichten. Das könnte zum Beispiel bedeuten, die "Segmentierung" und die Ausgestaltung der Dienstleistung stärker den Beteiligten zu überlassen, die etwa fallweise entscheiden würden, ob und wann eine Eingliederungsvereinbarung geschlossen wird. Solche Spielräume dürften aber nicht einseitig bei den Fachkräften liegen. Deren größerem Ermessensspielraum müssten dann Rechtsansprüche, Initiativ-, Wunschund Wahlrechte der Adressaten entsprechen, damit diese ihre Stärken in die Interaktion einbringen können. ${ }^{9}$

\subsection{AKTIVIERUNGSBEDARF - NICHT UNTERSTELLEN, ERMITTELN}

Die Annahme eines Aktivierungsdefizits trifft für einen großen Teil der Adressaten gar nicht und für andere unterschiedlich stark zu. Ob ein Bedarf an „aktivierender“ Fallbearbeitung besteht, wäre im Einzelfall zu ermitteln. Wie eine besondere, ,aktivierende" Variante der Dienstleistung aussehen könnte, lässt sich jedoch fachlich erst bestimmen, wenn unzureichende Suchaktivität nicht generell unterstellt wird. Wird dagegen der institutionelle Auftrag allein aus dem Aktivierungsdiskurs abgeleitet, so führt dies in vielen Fällen zu einer unbegründet hierarchischen Rollenverteilung zwischen „Aktivierern“, die gar nicht so viele zusätzliche Arbeitsmarktangebote einzubringen haben, und aktiven „Aktivierten“, die eher spezialisierte Unterstützung als umfassende Anleitung benötigen.

\subsection{AUF DEN NUTZEN FÜR DIE ADRESSATEN KOMMT ES AN}

Der personalisierte Auftrag unterstellt, dass die Dienstleistung den Adressaten einen Nutzen verspricht. Das anzuerkennen hieße, die Situation für die Fachkräfte zu normalisieren: Ein Risiko, dass die Klientel ausbleibt und das vom Leistungsträger ausgedachte Angebot nicht nützlich findet, trägt sonst jeder Anbieter sozialer Dienstleistungen, und Elemente von Wettbewerb werden an anderer Stelle oft gewünscht. Über den Nutzen dürften vor allem zwei Kriterien entscheiden: Es entspricht nicht der prekären materiellen Lebenslage der Adressaten, die existenzsichernden Geldleistungen nachrangig zu behandeln und damit zusammenhängende Probleme aus der individuellen Dienstleistung auszuschließen. Und: Im arbeitsmarktnahen
Kerngeschäft der Vermittlung müssten die Fachkräfte mehr Stellen- und Maßnahmeangebote anzubieten haben, um die Dienstleistung attraktiv zu machen.

Die Vorstellung von Fallmanagement als bloße Steuerung von Leistungen, die an spezialisierte Dritte delegiert werden, bewährt sich nicht. Aufträge an spezialisierte Dritte sind unvermeidlich, aber der pAp muss in vielen Bereichen eine entspezialisierte Erstversorgung selbst übernehmen, damit ein Problem bearbeitet werden kann.

\subsection{WIDERSTÄNDIGE POTENZIALE}

Die an Fürsorgetraditionen orientierten Regeln des Forderns und Förderns treffen auf Widerstand. Fachkräfte sind versucht, Anforderungen zu unterlaufen, indem sie z. B. Eingliederungsvereinbarungen zurückstellen, auf Sanktionen verzichten und den Adressaten Entscheidungen überlassen. Diese wiederum verteidigen Spielräume, betreiben Informationspolitik, verzichten gegebenenfalls auf Leistungen bzw. nehmen Sanktionen in Kauf, um Entscheidungsspielraum zu behalten. Die Konflikte hierüber werden wenig personalisiert ausgetragen. In den beobachteten Fällen kommen beide Seiten selbst im Konfliktfall noch „gut miteinander aus": Konflikte führten nicht zum Wunsch, die Ansprechperson zu wechseln. Selbst Sanktionsgespräche wurden freundlich geführt. Man braucht diese Widerstände nicht nur als Störungen aufzufassen. In ihnen zeigt sich auch, dass sich beide Seiten - Fachkräfte wie Adressaten - eben nicht an obrigkeitsstaatlichen oder paternalistischen Traditionsbeständen orientieren. Dies kann als Chance gelten, eine moderne arbeitsmarktnahe Dienstleistung auszugestalten, die auf sozialen Rechtsansprüchen beruht.

\section{9}

Sanktionen könnten darin bestehen, von besonderen Dienstleistungen auszuschließen, statt die existenzsichernden Leistungen zu kürzen. 
Autorenteam (2004): Fachkonzept „Beschäftigungsorientiertes Fallmanagement im SGB II“. Abschlussfassung des Arbeitskreises, Nürnberg Baethge-Kinsky, V./Bartelheimer, P./Henke, J./Land, R./Willisch, A./Wolf, A. (2006): Forschungsbericht, Neue soziale Dienstleistungen nach SGB II, Konzeptstudie (Typoskript), Göttingen

Bartelheimer, P. (2003): Case Management in der Praxis - Falldarstellungen und was man daraus lernen kann, in: Reis, C./Freyberg, T. von/ Kinstler, H.-J./Wende, L.: Pilotprojekt „Integrierte Hilfe zur Arbeit“ Endbericht der wissenschaftlichen Begleitung (im Auftrag des Ministeriums für Wirtschaft und Arbeit des Landes NRW), Frankfurt/M.,

S. 55-103

Berlit, U. (2006): Eingliederungsvereinbarungen nach dem SGB II Rechtsrahmen und Rechtsschutz; in: Sozialrecht aktuell 2, S. 41-50 Blien, U./Hirschenauer, F. (2005): Vergleichstypen 2005, Neufassung der Regionaltypisierung für Vergleiche zwischen Agenturbezirken, IAB-Forschungsbericht 24, Nürnberg

Bundesagentur für Arbeit (2006): Arbeitshilfe SGB II zur Eingliederungsvereinbarung (EinV) gem. § 15 SGB II (Stand: März 2006), Nürnberg Bundesministerium für Wirtschaft und Arbeit (BMWA) (2005): Evaluation der Grundsicherung für Arbeitsuchende im Rahmen der $\S \S 6 \mathrm{c}$ und 55 SGB II, Berlin

Deutscher Verein für öffentliche und private Fürsorge (2004): Empfehlungen des Deutschen Vereins zu Qualitätsstandards für das Fallmanagement, in: Nachrichtendienst des Deutschen Vereins für öffentliche und private Fürsorge 5, S. 149-153

Dunkel, W./Szymenderski, P./Voß, G. G. (2004): Dienstleistung als Interaktion - Ein Forschungsprojekt, in: Dunkel, W./Voß, G. G. (Hrsg.): Dienstleistung als Interaktion, Beiträge aus einem Forschungsprojekt Altenpflege - Deutsche Bahn - Call Center, München, S. 11-27
Göckler, R. (2006): Wo vollzieht sich Fördern und Fordern? Der Blick auf die Mitarbeiterlnnen, in: Beschäftigungspolitik: kommunal e. V. (bp:k) (Hrsg.): Netzwerk SGB II, Lokale Umsetzung des SGB II-Strukturen, Leistungsprozesse, Handlungsbedarfe, Fachtagung in Leipzig, 3./4. Mai 2006, Offenbach, S. 39-50

Harrach, E.-M. v./Loer, Th./Schmidtke, O. (2000): Verwaltung des Sozialen, Formen der subjektiven Bewältigung eines Strukturkonflikts, Konstanz

Hartz, P. (Hrsg.) (2002): Moderne Dienstleistungen am Arbeitsmarkt, Vorschläge der Kommission zum Abbau der Arbeitslosigkeit und zur Umstrukturierung der Bundesanstalt für Arbeit, Berlin

Kolbe, C./Reis, C. (2005): Vom Case Management zum „Fallmanagement" - Zur Praxis des Case Managements in der Sozialhilfe und der kommunalen Beschäftigungspolitik am Vorabend von Hartz IV, Frankfurt am Main

Reis, C. (2005): Leistungsprozesse im SGB II, Anregungen zur organisatorischen Ausgestaltung von Arbeitsgemeinschaften und "Optionskommunen“, Endbericht der wissenschaftlichen Begleitung zum Pilotprojekt "Arbeitsgemeinschaften" und Modellprojekt "JobCenter in Kreisen“", Teil A, Düsseldorf Reis, C. (2006): Wie kann das Fallmanagement in der Arbeitsvermittlung die Eigenverantwortung fördern?, in: WSI-Mitteilungen 4, S. 194-199 Wolf, K. (2006): Regionale Typisierung im SGB II, in: Bundesagentur für Arbeit: Jahresbericht 2005, SGB II, Grundsicherung für Arbeitsuchende, Nürnberg, S. 82-85, 103-111

Zeeb, M. (2006): Vermittlung, Bericht aus der Arbeitsgruppe, in: Pfannkuche, H./Lange, J. (Hrsg.): Und die Moral von der Geschicht'...? Die Evaluierung der Arbeitsmarktreformen ( „Hartz I bis III“"), Loccumer Protokolle 14, Loccum, S. 119-120 